\title{
STATIONARY SOLUTIONS FOR THE CAHN - HILLIARD EQUATION COUPLED WITH NEUMANN BOUNDARY CONDITIONS
}

\author{
I.B. Krasnyuk, Institute of Applied Mathematics and Mechanics of the NASU, \\ Donetsk, Ukraine, krasnjukigr@rambler.ru, \\ R.M. Taranets, University of California, Los Angeles, United States of America, \\ taranets_r@yahoo.com, \\ M. Chugunova, Claremont Graduate University, Claremont, United States of America, \\ marina.chugunova@cgu.edu
}

The structure of stationary states of the one-dimensional Cahn - Hilliard equation coupled with the Neumann boundary conditions has been studied. Here the free energy is given by a fourth order polynomial. The bifurcation diagram for existence and uniqueness of monotone solutions for this problem has been constructed. Namely, we find the length of the interval on which the solution monotonically increases or decreases and has one zero for some fixed values of physical parameters. Under the non-uniqueness we understand a possibility of existence of more than one monotone solutions for the same values of physical parameters.

Keywords: the Cahn - Hilliard equation; Neumann boundary conditions; steady states.

\section{Introduction}

We study the steady state solutions for the Cahn - Hilliard equation (see e.g. $[2,4,7,8]$ )

$$
u_{t}=\left(-\alpha u+\gamma u^{3}-u_{x x}\right)_{x x}, \quad 0<x<l, t>0,
$$

coupled with the Neumann boundary conditions

$$
u_{x}=u_{x x x}=0, \quad x=0, l,
$$

where $\alpha$ and $\gamma$ are some physical parameters. The authors of [2] have considered a structure of stationary solutions of problem (1) - (2) as a function of length $l$ and mass $m=\int_{0}^{l} u(s) d s$. They have counted the total number of monotone solutions, depending on the parameter values of the problem. In [2] phase portraits of the problem have been constructed which describe the monotone solutions for a special stored-energy function $f(u)+\frac{\varepsilon^{2}}{2}\left(u^{\prime}\right)^{2}$, where $\varepsilon$ is a small parameter. In this case, the function $f^{\prime}(u)$ has one negative minimum, one positive maximum, and $f( \pm \infty)= \pm \infty$.

In this paper, we study the following problem

$$
u^{\prime \prime}=-\alpha u+\gamma u^{3}-\sigma, \quad u^{\prime}(0)=u^{\prime}(l)=0,
$$

where $\sigma$ reflects influence of the average mass on qualitative behaviour of solutions; $\alpha$ is the main parameter of the problem because it is related to an interaction energy of the phase decomposition in a binary alloy; $\gamma$ is a parameter corresponding to thermodynamically stability of the system. In particular, $\alpha=\frac{E_{i n t}}{k_{B} T}\left(1-\frac{T}{T_{c}}\right)$, where $T_{c}$ is the critical temperature 
of the phase transition for order-disorder in a disordered binary alloy, which can be measured during the cooling process of the alloy; $E_{\text {int }}$ is the interaction energy between atoms of sorts $A$ and $B$ in the binary alloy. The energy functional corresponding to problem (3) can be written in the form

$$
E(u(t))=\int_{0}^{l}\left(\frac{1}{2}\left(u^{\prime}\right)^{2}+f(u)\right) d x, \quad E(u(t))=E(u(0))
$$

where the free energy $f(u)$ is

$$
f(u)=\frac{\gamma}{4} u^{4}-\frac{\alpha}{2} u^{2}-\sigma u+C
$$

Note that, in the case $\alpha>0, \gamma>0$, by the re-scaling $u=\sqrt{\frac{\alpha}{\gamma}} \hat{u}(\sqrt{\alpha} x)$, problem (3) can be reduced to

$$
\hat{u}^{\prime \prime}=-\hat{u}+\hat{u}^{3}-\hat{\sigma}, \quad \hat{u}^{\prime}(0)=\hat{u}^{\prime}(L)=0,
$$

where $L=\sqrt{\alpha} l$ and $\hat{\sigma}=\frac{\sigma}{\alpha} \sqrt{\frac{\gamma}{\alpha}}$. This case was studied in [2,7]. To the best of authors' knowledge all other cases were not considered in a literature. Therefore, in this article we investigate the existence of nontrivial solutions of problem (3) for all possible values of these parameters. In particular, we study an interesting case when $\gamma<0$. It should be mentioned that the case $\gamma<0, \alpha<0$ and $|\sigma|<\sigma_{0}:=\sqrt{\frac{4 \alpha^{3}}{27 \gamma}}$ leads to a non-uniqueness result (i.e. there exist two solutions with the same initial energy), depending on initial energy, but the solution of the problem is unique when $|\sigma| \rightarrow \sigma_{0}$. Also we describe all possible dynamical scenarios for the parameter values.

Moreover, our results can be applied to the Izing model. For example, the Izing model free energy in the vicinity of the phase transition may be written as the following (see, e.g. [6])

$$
f(u)=a+r u^{2}+s u^{4}+O\left(u^{6}\right) .
$$

In order for the system to be thermodynamically stable, the parameter on the highest even power of the order parameter must be positive. In this case, we show that $s>0$, hence the free energy is bounded. However, for the Neumann boundary value problem we can consider the case when the "open" system is thermodynamically unstable that corresponds to $s<0$. For example, in the case $s<0, r>0$ and $a \in\left[0, \frac{r}{2}\right)$, a solution is not unique. On the phase plane $u O u^{\prime}$, this phenomenon is illustrated by two loops with identical lengths of periods which are both symmetric with respect to the axis $O u$. Thus, there are two smooth nontrivial solutions.

\section{The Steady State Solutions for the Cahn - Hilliard Equation}

In this section, we find the existence interval, $L$ for a monotone solution by considering all possible values of the physical parameters. We proceed by examining all qualitatively different nine cases. Note that since all systems at hands are conservative they cannot have any attracting fixed points. Therefore their phase portraits can have only saddles and/or centers. 

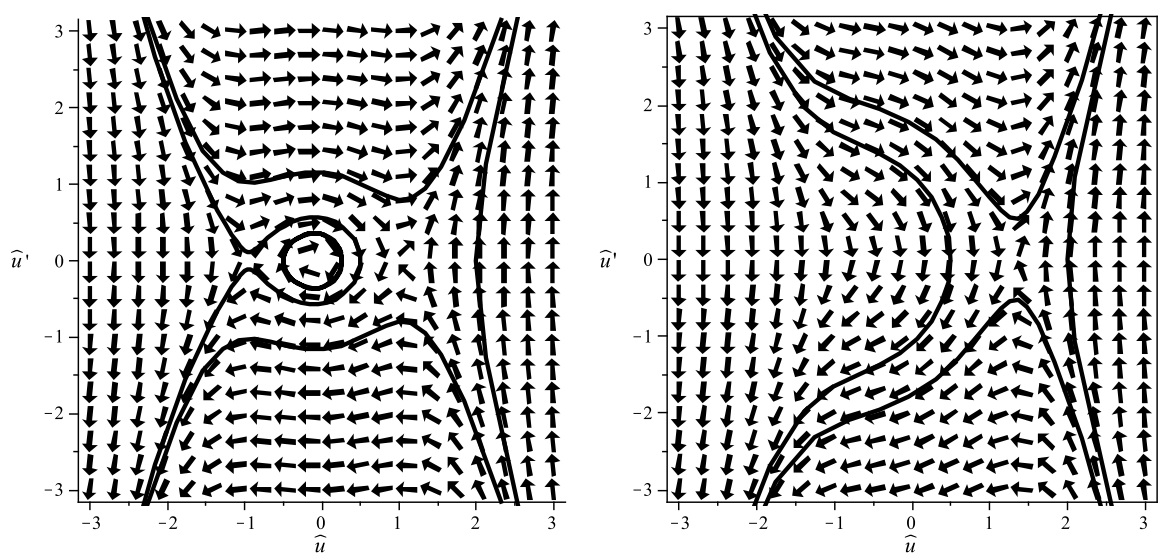

Fig 1. Phase diagram for the case $\alpha>0, \gamma>0$ with $\sigma=0,1$ on the left and $\sigma=1$ on the right

\subsection{Case $\alpha>0, \gamma>0$}

In this case, by the re-scaling $u=\sqrt{\frac{\alpha}{\gamma}} \hat{u}(\sqrt{\alpha} x)$, problem (3) can be reduced to

$$
\hat{u}^{\prime \prime}=-\hat{u}+\hat{u}^{3}-\hat{\sigma}, \quad \hat{u}^{\prime}(0)=\hat{u}^{\prime}(L)=0,
$$

where $L=\sqrt{\alpha} l$ and $\hat{\sigma}=\frac{\sigma}{\alpha} \sqrt{\frac{\gamma}{\alpha}}$. Integrating (6), we find that

$$
\frac{\left(\hat{u}^{\prime}\right)^{2}}{2}-\frac{\hat{u}^{4}}{4}+\frac{\hat{u}^{2}}{2}+\hat{\sigma} \hat{u}=p \Longleftrightarrow\left(\hat{u}^{\prime}\right)^{2}=\frac{1}{2}\left(\hat{u}^{4}-2 \hat{u}^{2}-4 \hat{\sigma} \hat{u}+4 p\right) .
$$

Note that the equation $\hat{u}^{3}-\hat{u}-\hat{\sigma}=0$ has

- three real roots

$$
\hat{u}_{k}=-\frac{2}{\sqrt{3}} \cos \left(\phi+\frac{2 k \pi}{3}\right), k=0,1,2, \quad \phi=\frac{1}{3} \arccos \left(\frac{3 \sqrt{3} \hat{\sigma}}{2}\right) ;
$$

provided that $\hat{\sigma}^{2}<\frac{4}{27}$ (two minimums and one maximum). Thus, we have two nontrivial solutions if $-\frac{\hat{u}_{\max }^{4}}{4}+\frac{\hat{u}_{\max }^{2}}{2}+\hat{\sigma} \hat{u}_{\max } \leq p<-\frac{\hat{u}_{\min }^{4}}{4}+\frac{\hat{u}_{\min }^{2}}{2}+\hat{\sigma} \hat{u}_{\text {min }}$ and the lengths of intervals, where the corresponding solution has only one zero, are

$$
L_{0,1}=\sqrt{2} \int_{u_{1}}^{u_{2}} \frac{d t}{\sqrt{t^{4}-2 t^{2}-4 \hat{\sigma} t+4 p}}, \quad L_{0,2}=\sqrt{2} \int_{u_{3}}^{u_{4}} \frac{d t}{\sqrt{t^{4}-2 t^{2}-4 \hat{\sigma} t+4 p}}
$$

where $u_{k}$ are four real roots of the equation $t^{4}-2 t^{2}-4 \hat{\sigma} t+4 p=0$ such that $u_{1}<u_{2} \leq$ $u_{3}<u_{4}$. We have only one nontrivial solution if $p<-\frac{\hat{u}_{\max }^{4}}{4}+\frac{\hat{u}_{\max }^{2}}{2}+\hat{\sigma} \hat{u}_{\max }$ with the length of interval, where the solution has only one zero, given by

$$
L_{0,3}=\sqrt{2} \int_{u_{1}}^{u_{2}} \frac{d t}{\sqrt{t^{4}-2 t^{2}-4 \hat{\sigma} t+4 p}},
$$



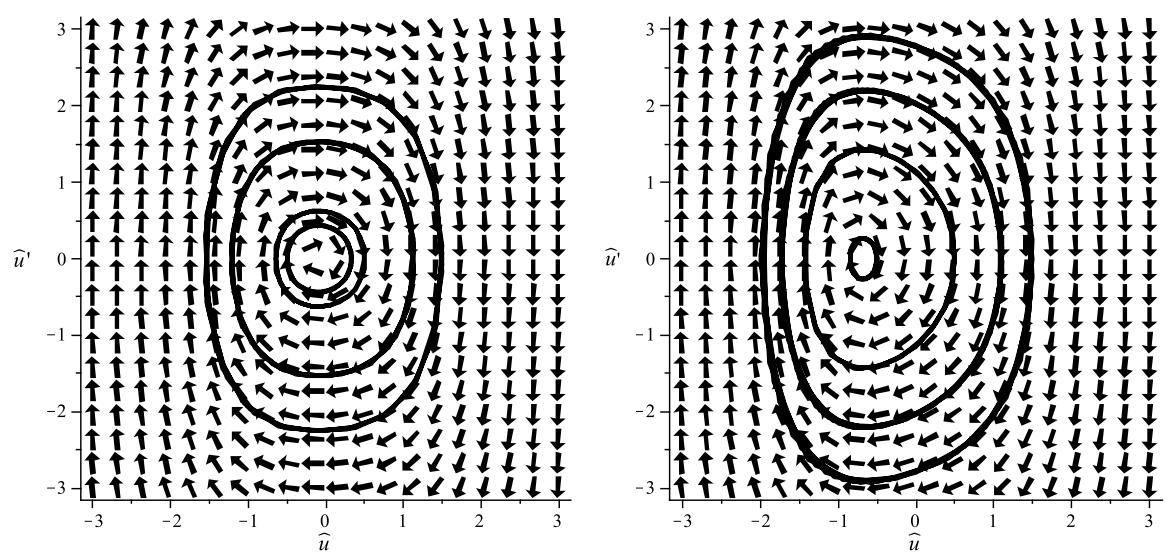

Fig 2. Phase diagram for the case $\alpha>0, \gamma<0$ with $\sigma=0,1$ on the left and $\sigma=1$ on the right

where $u_{k}$ are two real roots of the equation $t^{4}-2 t^{2}-4 \hat{\sigma} t+4 p=0$ such that $u_{1}<u_{2}$;

- one real root

$$
\hat{u}_{\min }=\left(\frac{\hat{\sigma}}{2}+\sqrt{\frac{\hat{\sigma}^{2}}{4}-\frac{1}{27}}\right)^{1 / 3}+\left(\frac{\hat{\sigma}}{2}-\sqrt{\frac{\hat{\sigma}^{2}}{4}-\frac{1}{27}}\right)^{1 / 3}
$$

provided that $\hat{\sigma}^{2}>\frac{4}{27}$ (one minimum). Thus, we have a nontrivial solution if $p<-\frac{\hat{u}_{\min }^{4}}{4}+$ $\frac{\hat{u}_{\min }^{2}}{2}+\hat{\sigma} \hat{u}_{\min }$ with the length of interval, where the solution has only one zero, given by

$$
L_{0}=\sqrt{2} \int_{u_{1}}^{u_{2}} \frac{d t}{\sqrt{t^{4}-2 t^{2}-4 \hat{\sigma} t+4 p}},
$$

where $u_{k}$ are two real roots of the equation $t^{4}-2 t^{2}-4 \hat{\sigma} t+4 p=0$ such that $u_{1}<u_{2}$;

- two real roots

$$
\hat{u}_{\text {min }}=2\left(\frac{\hat{\sigma}}{2}\right)^{1 / 3}, \quad \hat{u}_{f l}=-\left(\frac{\hat{\sigma}}{2}\right)^{1 / 3}
$$

provided that $\hat{\sigma}^{2}=\frac{4}{27}$ (one minimum). Thus, we have a nontrivial solution if $p<-\frac{\hat{u}_{\min }^{4}}{4}+$ $\frac{\hat{u}_{\min }^{2}}{2}+\hat{\sigma} \hat{u}_{\min }=2\left(\frac{\hat{\sigma}}{2}\right)^{2 / 3}$ with the length of interval, where the solution has only one zero, given by

$$
L_{0}=\sqrt{2} \int_{u_{1}}^{u_{2}} \frac{d t}{\sqrt{t^{4}-2 t^{2}-4 \hat{\sigma} t+4 p}},
$$

where $u_{k}$ are the two real roots of the equation $t^{4}-2 t^{2}+4 \hat{\sigma} t-4 p=0$ such that $u_{1}<u_{2}$.

Thus, if $0<L<L_{0}$ then the corresponding solution of the problem has no zeros located on the interval $(0, L)$. If $k L_{0} \leq L<(k+1) L_{0}$, where $k=1,2, \ldots$, then the solution has exactly $k$ zeros located on the interval $(0, L)$.

\subsection{Case $\alpha>0, \gamma<0$}

In this case, by the re-scaling $u=\sqrt{-\frac{\alpha}{\gamma}} \hat{u}(\sqrt{\alpha} x)$, problem $(3)$ can be reduced to

$$
\hat{u}^{\prime \prime}=-\hat{u}-\hat{u}^{3}-\hat{\sigma}, \quad \hat{u}^{\prime}(0)=\hat{u}^{\prime}(L)=0,
$$


where $L=\sqrt{\alpha} l$ and $\hat{\sigma}=\frac{\sigma}{\alpha} \sqrt{-\frac{\gamma}{\alpha}}$. Integrating (11), we obtain

$$
\frac{\left(\hat{u}^{\prime}\right)^{2}}{2}+\frac{\hat{u}^{4}}{4}+\frac{\hat{u}^{2}}{2}+\hat{\sigma} \hat{u}=p \Longleftrightarrow\left(\hat{u}^{\prime}\right)^{2}=\frac{1}{2}\left(-\hat{u}^{4}-2 \hat{u}^{2}-4 \hat{\sigma} \hat{u}+4 p\right) .
$$

Note that the equation $\hat{u}^{3}+\hat{u}+\hat{\sigma}=0$ has only one real root

$$
\hat{u}_{\max }=\left(-\frac{\hat{\sigma}}{2}+\sqrt{\frac{\hat{\sigma}^{2}}{4}+\frac{1}{27}}\right)^{1 / 3}+\left(-\frac{\hat{\sigma}}{2}-\sqrt{\frac{\hat{\sigma}^{2}}{4}+\frac{1}{27}}\right)^{1 / 3}
$$

for any $\hat{\sigma} \in R^{1}$ (one maximum). Thus, we have a nontrivial solution if $p>\frac{\hat{u}_{\max }^{4}}{4}+\frac{\hat{u}_{\max }^{2}}{2}+$ $\hat{\sigma} \hat{u}_{\max }$ with the length of interval, where the solution has only one zero, given by

$$
L_{0}=\sqrt{2} \int_{u_{1}}^{u_{2}} \frac{d t}{\sqrt{-t^{4}-2 t^{2}-4 \hat{\sigma} t+4 p}},
$$

where $u_{k}$ are two real roots of the equation $t^{4}+2 t^{2}+4 \hat{\sigma} t-4 p=0$ such that $u_{1}<u_{2}$. For example, if $\hat{\sigma}=0$ and $p>0$ then we have

$$
\frac{\left(u^{\prime}\right)^{2}}{A^{2}}+\frac{\left(u^{2}+1\right)^{2}}{B^{4}}=1
$$

where $A^{2}=\frac{4 p+1}{2}, B^{4}=4 p+1$, and from (13) it follows that

$$
L_{0}=\frac{B^{2}}{A} \int_{-\sqrt{B^{2}-1}}^{\sqrt{B^{2}-1}} \frac{d t}{\sqrt{B^{4}-\left(t^{2}+1\right)^{2}}}=\tilde{A} \int_{-1}^{1} \frac{d t}{\sqrt{\left(1-t^{2}\right)\left(1+k^{2} t^{2}\right)}}
$$

where

$$
\tilde{A}=\frac{B^{2}}{A \sqrt{B^{2}+1}}, \quad k^{2}=\frac{B^{2}-1}{B^{2}+1} \in(0,1) .
$$

Let $t=\sin \varphi$ in integral (14). Then

$$
L_{0}=2 \tilde{A} \int_{0}^{\pi / 2} \frac{d \varphi}{\sqrt{1+k^{2} \sin ^{2} \varphi}}, \quad L_{0} \in(2.622 \tilde{A}, \pi \tilde{A}) .
$$

1.3. Case $\alpha<0, \gamma>0$

In this case, by the re-scaling $u=\sqrt{-\frac{\alpha}{\gamma}} \hat{u}(\sqrt{-\alpha} x)$, problem (3) can be reduced to

$$
\hat{u}^{\prime \prime}=\hat{u}+\hat{u}^{3}-\hat{\sigma}, \quad \hat{u}^{\prime}(0)=\hat{u}^{\prime}(L)=0,
$$

where $L=\sqrt{-\alpha} l$ and $\hat{\sigma}=-\frac{\sigma}{\alpha} \sqrt{-\frac{\gamma}{\alpha}}$. Integrating (17), we obtain

$$
\frac{\left(\hat{u}^{\prime}\right)^{2}}{2}-\frac{\hat{u}^{4}}{4}-\frac{\hat{u}^{2}}{2}+\hat{\sigma} \hat{u}=p \Longleftrightarrow\left(\hat{u}^{\prime}\right)^{2}=\frac{1}{2}\left(\hat{u}^{4}+2 \hat{u}^{2}-4 \hat{\sigma} \hat{u}+4 p\right) .
$$

Note that the equation $\hat{u}^{3}+\hat{u}-\hat{\sigma}=0$ has only one real root

$$
\hat{u}_{\min }=\left(\frac{\hat{\sigma}}{2}+\sqrt{\frac{\hat{\sigma}^{2}}{4}+\frac{1}{27}}\right)^{1 / 3}+\left(\frac{\hat{\sigma}}{2}-\sqrt{\frac{\hat{\sigma}^{2}}{4}+\frac{1}{27}}\right)^{1 / 3}
$$



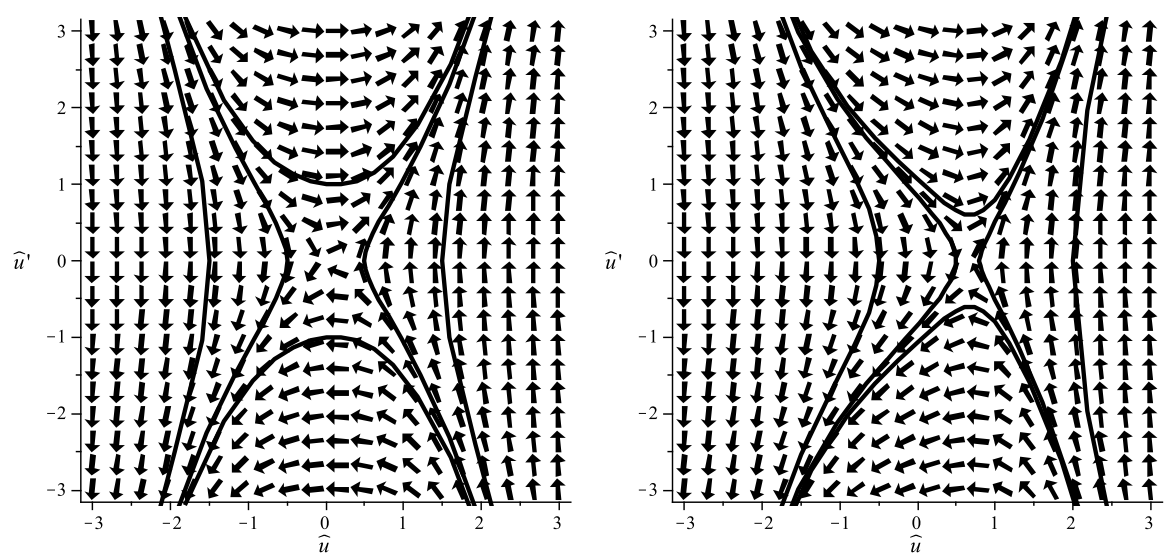

Fig 3. Phase diagram for the case $\alpha<0, \gamma>0$ with $\sigma=0,1$ on the left and $\sigma=1$ on the right
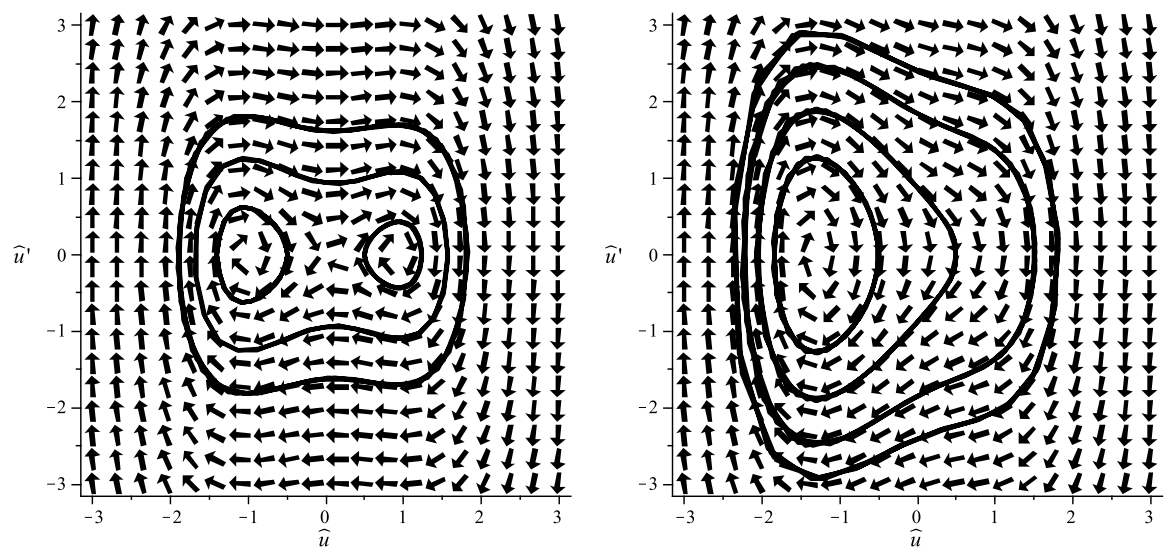

Fig 4. Phase diagram for the case $\alpha<0, \gamma<0$ with $\sigma=0,1$ on the left and $\sigma=1$ on the right

for any $\hat{\sigma} \in R^{1}$ (one minimum). Thus, we have a nontrivial solution if $p>-\frac{\hat{u}_{\min }^{4}}{4}-\frac{\hat{u}_{\min }^{2}}{2}+$ $\hat{\sigma} \hat{u}_{\text {min }}$ with the length of interval, where the solution has only one zero, given by

$$
L_{0}=\sqrt{2} \int_{u_{1}}^{u_{2}} \frac{d t}{\sqrt{t^{4}+2 t^{2}-4 \hat{\sigma} t+4 p}}
$$

where $u_{k}$ are two real roots of the equation $t^{4}+2 t^{2}-4 \hat{\sigma} t+4 p=0$ such that $u_{1}<u_{2}$.

1.4. Case $\alpha<0, \gamma<0$

In this case, by the re-scaling $u=\sqrt{\frac{\alpha}{\gamma}} \hat{u}(\sqrt{-\alpha} x)$, problem (3) can be reduced to

$$
\hat{u}^{\prime \prime}=\hat{u}-\hat{u}^{3}-\hat{\sigma}, \quad \hat{u}^{\prime}(0)=\hat{u}^{\prime}(L)=0,
$$


where $L=\sqrt{-\alpha} l$ and $\hat{\sigma}=-\frac{\sigma}{\alpha} \sqrt{\frac{\gamma}{\alpha}}$. Integrating (19), we find that

$$
\frac{\left(\hat{u}^{\prime}\right)^{2}}{2}+\frac{\hat{u}^{4}}{4}-\frac{\hat{u}^{2}}{2}+\hat{\sigma} \hat{u}=p \Longleftrightarrow\left(\hat{u}^{\prime}\right)^{2}=\frac{1}{2}\left(-\hat{u}^{4}+2 \hat{u}^{2}-4 \hat{\sigma} \hat{u}+4 p\right) .
$$

Note that the equation $-\hat{u}^{3}+\hat{u}-\hat{\sigma}=0$ has

- three real roots

$$
\hat{u}_{k}=-\frac{2}{\sqrt{3}} \cos \left(\phi+\frac{2 k \pi}{3}\right), k=0,1,2, \quad \phi=\frac{1}{3} \arccos \left(\frac{3 \sqrt{3} \hat{\sigma}}{2}\right) ;
$$

provided that $\hat{\sigma}^{2}<\frac{4}{27}$ (two maximums and one minimum). Thus, we have two nontrivial solutions if $\frac{\hat{u}_{\max }^{4}}{4}-\frac{\hat{u}_{\max }^{2}}{2}+\hat{\sigma} \hat{u}_{\max }<p \leq \frac{\hat{u}_{\min }^{4}}{4}-\frac{\hat{u}_{\min }^{2}}{2}+\hat{\sigma} \hat{u}_{\min }$ with the lengths of intervals, where the corresponding solution has only one zero, given by

$$
L_{0,1}=\sqrt{2} \int_{u_{1}}^{u_{2}} \frac{d t}{\sqrt{-t^{4}+2 t^{2}-4 \hat{\sigma} t+4 p}}, \quad L_{0,2}=\sqrt{2} \int_{u_{3}}^{u_{4}} \frac{d t}{\sqrt{-t^{4}+2 t^{2}-4 \hat{\sigma} t+4 p}}
$$

where $u_{k}$ are four real roots of the equation $t^{4}-2 t^{2}+4 \hat{\sigma} t-4 p=0$ such that $u_{1}<u_{2}<$ $u_{3}<u_{4}$.

We have only one nontrivial solution if $p>\frac{\hat{u}_{\min }^{4}}{4}-\frac{\hat{u}_{\min }^{2}}{2}+\hat{\sigma} \hat{u}_{\min }$ with the length of interval, where the solution has only one zero, given by

$$
L_{0,3}=\sqrt{2} \int_{u_{1}}^{u_{2}} \frac{d t}{\sqrt{-t^{4}+2 t^{2}-4 \hat{\sigma} t+4 p}}
$$

where $u_{k}$ are two real roots of the equation $t^{4}-2 t^{2}+4 \hat{\sigma} t-4 p=0$ such that $u_{1}<u_{2}$;

- one real root

$$
\hat{u}_{\max }=\left(-\frac{\hat{\sigma}}{2}+\sqrt{\frac{\hat{\sigma}^{2}}{4}-\frac{1}{27}}\right)^{1 / 3}+\left(-\frac{\hat{\sigma}}{2}-\sqrt{\frac{\hat{\sigma}^{2}}{4}-\frac{1}{27}}\right)^{1 / 3}
$$

provided that $\hat{\sigma}^{2}>\frac{4}{27}$ (one maximum). Thus, we have a nontrivial solution if $p>\frac{\hat{u}_{\max }^{4}}{4}-$ $\frac{\hat{u}_{\max }^{2}}{2}+\hat{\sigma} \hat{u}_{\max }$ with the length of interval, where the solution has only one zero, given by

$$
L_{0}=\sqrt{2} \int_{u_{1}}^{u_{2}} \frac{d t}{\sqrt{-t^{4}+2 t^{2}-4 \hat{\sigma} t+4 p}}
$$

where $u_{k}$ are two real roots of the equation $t^{4}-2 t^{2}+4 \hat{\sigma} t-4 p=0$ such that $u_{1}<u_{2}$;

- two real roots

$$
\hat{u}_{\max }=-2\left(\frac{\hat{\sigma}}{2}\right)^{1 / 3}, \quad \hat{u}_{f l}=\left(\frac{\hat{\sigma}}{2}\right)^{1 / 3}
$$

provided that $\hat{\sigma}^{2}=\frac{4}{27}$ (one maximum). Thus, we have a nontrivial solution if $p>\frac{\hat{u}_{\max }^{4}}{4}-$ $\frac{\hat{u}_{\max }^{2}}{2}+\hat{\sigma} \hat{u}_{\max }=-2\left(\frac{\hat{\sigma}}{2}\right)^{2 / 3}$ with the length of interval, where the solution has only one zero, given by

$$
L_{0}=\sqrt{2} \int_{u_{1}}^{u_{2}} \frac{d t}{\sqrt{-t^{4}+2 t^{2}-4 \hat{\sigma} t+4 p}}
$$



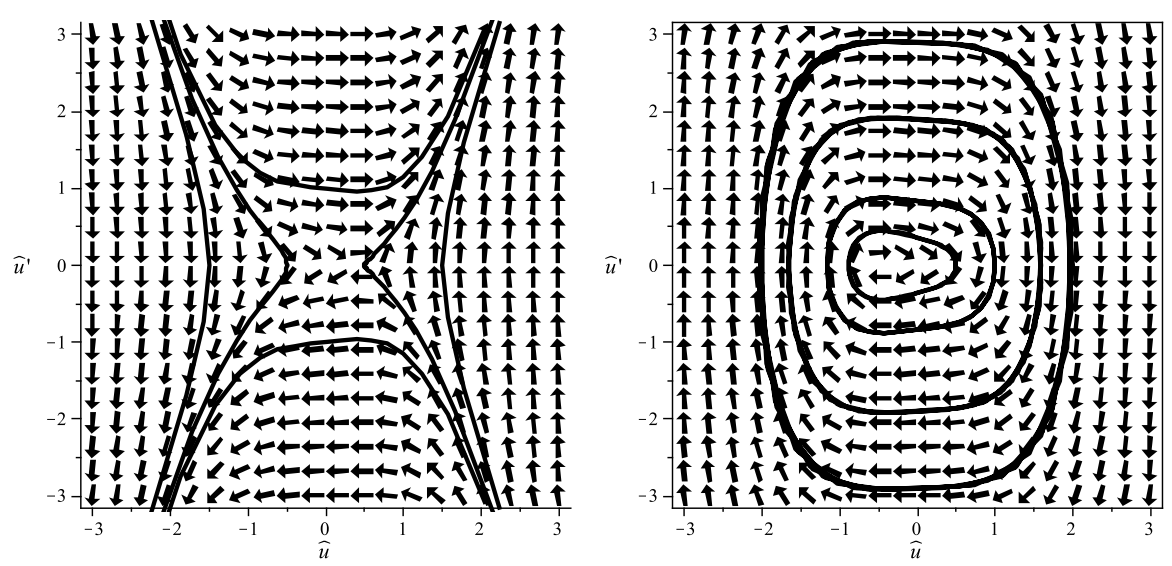

Fig 5. Phase diagram for the case $\alpha=0, \gamma>0$ with $\sigma=0,1$ on the left and for the case $\alpha=0, \gamma<0$ with $\sigma=0,1$ on the right

where $u_{k}$ are two real roots of the equation $t^{4}-2 t^{2}+4 \hat{\sigma} t-4 p=0$ such that $u_{1}<u_{2}$.

For example, if $\hat{\sigma}=0$ and $p>0$ then we have

$$
\frac{\left(u^{\prime}\right)^{2}}{A^{2}}+\frac{\left(u^{2}-1\right)^{2}}{B^{4}}=1
$$

where $A^{2}=\frac{4 p+1}{2}, B^{4}=4 p+1$, and from (24) it follows that

$$
L_{0}=\frac{B^{2}}{A} \int_{-\sqrt{B^{2}+1}}^{\sqrt{B^{2}+1}} \frac{d t}{\sqrt{B^{4}-\left(t^{2}-1\right)^{2}}}=\tilde{A} \int_{-1}^{1} \frac{d t}{\sqrt{\left(1-t^{2}\right)\left(1+k^{2} t^{2}\right)}}
$$

where

$$
\tilde{A}=\frac{B^{2}}{A \sqrt{B^{2}-1}}, \quad k^{2}=\frac{B^{2}+1}{B^{2}-1}>1 .
$$

Let $t=\sin \varphi$ in integral (25). Then

$$
L_{0}=2 \tilde{A} \int_{0}^{\pi / 2} \frac{d \varphi}{\sqrt{1+k^{2} \sin ^{2} \varphi}}<\pi \tilde{A} .
$$

1.5. Case $\alpha=0, \gamma \neq 0$

If $\alpha=0, \gamma>0$, by the re-scaling $u=\hat{u}(\sqrt{\gamma} x)$, then problem (3) can be reduced to the one

$$
\hat{u}^{\prime \prime}=\hat{u}^{3}-\hat{\sigma}, \quad \hat{u}^{\prime}(0)=\hat{u}^{\prime}(L)=0,
$$

where $L=\sqrt{\gamma} l$ and $\hat{\sigma}=-\frac{\sigma}{\gamma}$. Integrating (28), we find that

$$
\frac{\left(\hat{u}^{\prime}\right)^{2}}{2}-\frac{\hat{u}^{4}}{4}+\hat{\sigma} \hat{u}=p \Longleftrightarrow\left(\hat{u}^{\prime}\right)^{2}=\frac{1}{2}\left(\hat{u}^{4}-4 \hat{\sigma} \hat{u}+4 p\right) .
$$


Thus, problem (28) has a nontrivial solution provided that $p>\frac{3}{4} \hat{\sigma}^{4 / 3}$, i. e. $p>\frac{3}{4}\left(\frac{\sigma}{\gamma}\right)^{4 / 3}$. The length of interval, where the solution has only one zero, is

$$
L_{0}=\sqrt{2} \int_{u_{1}}^{u_{2}} \frac{d t}{\sqrt{t^{4}-4 \hat{\sigma} t+4 p}}
$$

where $u_{k}$ are two real roots of the equation $t^{4}-4 \hat{\sigma} t+4 p=0$.

If $\alpha=0, \gamma<0$, by the re-scaling $u=\hat{u}(\sqrt{-\gamma} x)$, then problem (3) can be reduced to

$$
\hat{u}^{\prime \prime}=-\hat{u}^{3}-\hat{\sigma}, \quad \hat{u}^{\prime}(0)=\hat{u}^{\prime}(L)=0,
$$

where $L=\sqrt{-\gamma} l$ and $\hat{\sigma}=\frac{\sigma}{\gamma}$. Integrating (30), we find that

$$
\frac{\left(\hat{u}^{\prime}\right)^{2}}{2}+\frac{\hat{u}^{4}}{4}+\hat{\sigma} \hat{u}=p \Longleftrightarrow\left(\hat{u}^{\prime}\right)^{2}=\frac{1}{2}\left(-\hat{u}^{4}-4 \hat{\sigma} \hat{u}+4 p\right) .
$$

Thus, problem (30) has a nontrivial solution provided that $p>-\frac{3}{4} \hat{\sigma}^{4 / 3}$, i. e. $p>-\frac{3}{4}\left(\frac{\sigma}{\gamma}\right)^{4 / 3}$. The length of interval, where the solution has only one zero, is

$$
L_{0}=\sqrt{2} \int_{u_{1}}^{u_{2}} \frac{d t}{\sqrt{-t^{4}-4 \hat{\sigma} t+4 p}}
$$

where $u_{k}$ are two real roots of the equation $t^{4}+4 \hat{\sigma} t-4 p=0$. For example, if $\hat{\sigma}=0$ and $p>0$ then we have the curve

$$
\frac{\left(\hat{u}^{\prime}\right)^{2}}{A^{2}}+\frac{\hat{u}^{4}}{B^{4}}=1
$$

where $A^{2}=2 p, B^{4}=4 p$. Obviously, the length of interval, where the solution has only one zero, is

$$
L_{0}=\frac{B^{2}}{A} \int_{-B}^{B} \frac{d t}{\sqrt{B^{4}-t^{4}}}=\frac{2 B}{A} \int_{0}^{1} \frac{d t}{\sqrt{1-t^{4}}}=\frac{B}{2 A} \mathbf{B}\left(\frac{1}{4}, \frac{1}{2}\right) \approx 2,622 \frac{B}{A}=\frac{2,622}{p^{\frac{1}{4}}},
$$

where $\mathbf{B}(a, b):=\int_{0}^{1} x^{a-1}(1-x)^{b-1} d x$ is the beta function.

1.6. Case $\gamma=0, \alpha \in R^{1}$

If $\alpha=0, \gamma=0$ then problem (3) can be reduced to

$$
u^{\prime \prime}=-\sigma, \quad u^{\prime}(0)=u^{\prime}(l)=0 .
$$

This problem has the following solution

$$
u(x)=C \quad \forall C \in R^{1} \text { and } \sigma=0,
$$

and it has no solutions if $\sigma \neq 0$. 

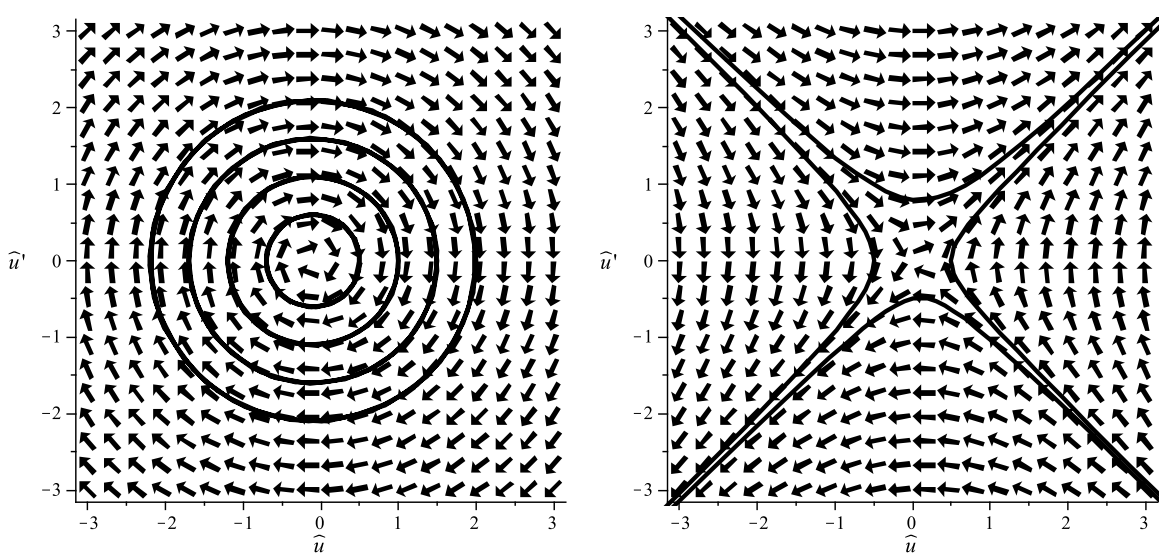

Fig 6. Phase diagram for the case $\alpha>0, \gamma=0$ with $\sigma=0,1$ on the left and for the case $\alpha<0, \gamma=0$ with $\sigma=0,1$ on the right

If $\alpha>0, \gamma=0$, by the re-scaling $u=\hat{u}(\sqrt{\alpha} x)$, problem (3) can be reduced to

$$
\hat{u}^{\prime \prime}=-\hat{u}-\hat{\sigma}, \quad \hat{u}^{\prime}(0)=\hat{u}^{\prime}(L)=0,
$$

where $L=\sqrt{\alpha} l$ and $\hat{\sigma}=\frac{\sigma}{\alpha}$. This problem has the following solution

$$
u(x)=C \cos (\sqrt{\alpha} x)-\hat{\sigma}, l=\frac{\pi k}{\sqrt{\alpha}}, k \in Z^{+}, C \in R^{1} .
$$

If $\alpha<0, \gamma=0$, by the re-scaling $u=\hat{u}(\sqrt{-\alpha} x)$, problem (3) can be reduced to

$$
\hat{u}^{\prime \prime}=\hat{u}-\hat{\sigma}, \quad \hat{u}^{\prime}(0)=\hat{u}^{\prime}(L)=0,
$$

where $L=\sqrt{-\alpha} l$ and $\hat{\sigma}=-\frac{\sigma}{\alpha}$. This problem has the following solution

$$
u(x)=\hat{\sigma} .
$$

For convenience, we summarize our existence results in the form of Table.

\subsection{Example}

As shown in [3], the possible dimensionless function $f(u, T)$ at fixed $T$ can be written as

$$
f(u, T)=\frac{T}{4 T_{0}}\left(1-u^{2}\right)^{2}+a \frac{T-T_{0}}{T_{0}}(u+1)^{2}+\frac{c}{f_{0}} T \ln \left(\frac{T}{T_{0}}\right),
$$

where $T_{0}$ is a critical temperature, $c$ is the heat capacity, $a=\frac{\ell}{4 f_{0}}$ is dimensionless, $\ell$ is the latent heat, $f_{0}$ is a parameter with dimensions of energy density. Comparing (37) with our $f(u)$, we find that

$$
\begin{gathered}
\gamma=\frac{T}{T_{0}}, \alpha=\frac{T-2 a\left(T-T_{0}\right)}{T_{0}}, \sigma=\frac{2 a\left(T_{0}-T\right)}{T_{0}}, \\
C=\frac{c}{f_{0}} T \ln \left(\frac{T}{T_{0}}\right)+\frac{T+4 a\left(T-T_{0}\right)}{4 T_{0}} .
\end{gathered}
$$




\section{Table}

Conditions on the problem's parameters for existence and non-existence of nontrivial solutions

\begin{tabular}{|c|c|c|c|c|}
\hline$\alpha$ & $\sigma$ & $\gamma$ & $\mathrm{p}$ & $L_{0}$ \\
\hline$>0$ & $\sigma^{2}<\frac{4 \alpha^{3}}{27 \gamma}$ & $>0$ & $p \in\left[p_{\min }, p_{\max }\right)$ & Yes $(2$ sol's $)$ \\
$>0$ & $\sigma^{2}<\frac{4 \alpha^{3}}{27 \gamma}$ & $>0$ & $p<p_{\min }$ & Yes \\
$>0$ & $\sigma^{2}<\frac{4 \alpha^{3}}{27 \gamma}$ & $>0$ & $p \geq p_{\max }$ & No \\
$>0$ & $\sigma^{2} \geq \frac{4 \alpha^{3}}{27 \gamma}$ & $>0$ & $p<p_{\min }$ & Yes \\
$>0$ & $\sigma^{2} \geq \frac{4 \alpha^{3}}{27 \gamma}$ & $>0$ & $p \geq p_{\min }$ & No \\
$>0$ & $R^{1}$ & $<0$ & $p>p_{\min }$ & Yes \\
$>0$ & $R^{1}$ & $<0$ & $p \leq p_{\min }$ & No \\
$>0$ & $R^{1}$ & $=0$ & $R^{1}$ & Yes (family sol's $)$ \\
\hline$<0$ & $R^{1}$ & $>0$ & $p>p_{\min }$ & Yes \\
$<0$ & $R^{1}$ & $>0$ & $p \leq p_{\min }$ & No \\
$<0$ & $\sigma^{2}<\frac{4 \alpha^{3}}{27 \gamma}$ & $<0$ & $p \in\left(p_{\min }, p_{\max }\right]$ & Yes $(2$ sol's $)$ \\
$<0$ & $\sigma^{2}<\frac{4 \alpha^{3}}{27 \gamma}$ & $<0$ & $p>p_{\max }$ & Yes \\
$<0$ & $\sigma^{2}<\frac{4 \alpha^{3}}{27 \gamma}$ & $<0$ & $p \leq p_{\min }$ & No \\
$<0$ & $\sigma^{2} \geq \frac{4 \alpha^{3}}{27 \gamma}$ & $<0$ & $p>p_{\min }$ & Yes \\
$<0$ & $\sigma^{2} \geq \frac{4 \alpha^{3}}{27 \gamma}$ & $<0$ & $p \leq p_{\min }$ & No \\
$<0$ & $R^{1}$ & $=0$ & $R^{1}$ & No \\
\hline$=0$ & $R^{1}$ & $>0$ & $p>\frac{3}{4}\left(\frac{\sigma}{\gamma}\right)^{4 / 3}$ & Yes \\
$=0$ & $R^{1}$ & $>0$ & $p \leq \frac{3}{4}\left(\frac{\sigma}{\gamma}\right)^{4 / 3}$ & No \\
$=0$ & $R^{1}$ & $<0$ & $p>-\frac{3}{4}\left(\frac{\sigma}{\gamma}\right)^{4 / 3}$ & Yes \\
$=0$ & $R^{1}$ & $<0$ & $p \leq \frac{3}{4}\left(\frac{\sigma}{\gamma}\right)^{4 / 3}$ & No \\
$=0$ & $R^{1}$ & $=0$ & $R^{1}$ & No \\
\hline
\end{tabular}

For example, if $0<T \leq T_{0}$ then $\alpha>0, \gamma>0$, and $\sigma \geq 0$. In this case, according to the paragraph 2.1, problem (3) has nontrivial solutions. On the other hand, if $T>T_{0}$ and $a<\frac{T}{2\left(T-T_{0}\right)}$ then $\alpha>0, \gamma>0, \sigma<0$ and we again have the existence of nontrivial solutions.

\section{The Steady States for General Free Energy}

In the previous section, we have studied the case when the free energy contains the linear term but it has no cubic term. Note that the presence of a linear term breaks down the symmetry of the free energy. Next, we will examine how the length of the existence interval can be effected by the cubic term. It is possible to introduce asymmetry in a phase diagram by adding odd powers to the free energy expansion so that

$$
f(u, T)=a_{0}(T)+a_{1}(T) u+a_{2}(T) \frac{u^{2}}{2}+a_{3}(T) \frac{u^{3}}{3}+a_{4}(T) \frac{u^{4}}{4}+O\left(u^{5}\right) .
$$


Note that the special case $a_{1}(T)=a_{3}(T)=0$ was introduced in $[6$, p. 19, (2.41)]. The authors assumed that $a_{4}(T)>0$ and $a_{2}(T)<0$. Then $f(u, T)$ has maximum at $u=0$ and minimum at $u_{ \pm}= \pm \sqrt{-\frac{a_{2}}{a_{4}}}$. By definition, it is easy to verify that $f(0, T)=a_{0}(T)>0$ and $f\left(u_{ \pm}, T\right)<0$ as $a_{2}^{2}>4 a_{0} a_{4}>0$. It means that there is a point $u_{0} \in\left(0, \sqrt{-\frac{a_{2}}{a_{4}}}\right)$ such that $f\left( \pm u_{0}, T\right)=0$. Thus, we can calculate the minimal length as

$$
L_{0}=\sqrt{\frac{2}{a_{4}}} \int_{-u_{0}}^{u_{0}} \frac{d t}{\sqrt{\left(t^{2}-u_{0}^{2}\right)\left(t^{2}-u_{1}^{2}\right)}}=\sqrt{\frac{2}{a_{4} u_{1}^{2}}} \int_{-1}^{1} \frac{d t}{\sqrt{\left(1-t^{2}\right)\left(1-k^{2} t^{2}\right)}},
$$

where

$$
u_{0}=\sqrt{-\frac{a_{2}}{a_{4}}-\frac{1}{a_{4}} \sqrt{a_{2}^{2}-4 a_{0} a_{4}}}, \quad u_{1}=\sqrt{-\frac{a_{2}}{a_{4}}+\frac{1}{a_{4}} \sqrt{a_{2}^{2}-4 a_{0} a_{4}}}, k^{2}=\frac{u_{0}^{2}}{u_{1}^{2}} \in(0,1) .
$$

Substituting the new variable $t=\sin \varphi$ into (39), we have

$$
L_{0}=2 \sqrt{\frac{2}{a_{4} u_{1}^{2}}} \int_{0}^{\pi / 2} \frac{d \varphi}{\sqrt{1-k^{2} \sin ^{2} \varphi}} .
$$

Next, consider the equation

$$
\frac{1}{2}\left(u^{\prime}\right)^{2}=R(u)
$$

then

$$
L_{0}=\frac{\sqrt{2}}{2} \int_{u_{1}}^{u_{2}} \frac{d z}{\sqrt{R(z)}}
$$

where $u_{i}$ are real roots of $R(u)=0$ such that $u_{1}<u_{2}$. Here

$$
R(z)=a z^{4}+b z^{3}+c z^{2}+d z+e=a\left(z^{2}+p z+q\right)\left(z^{2}+p^{\prime} z+q^{\prime}\right)
$$

where $a, b, c, d, e, p, q, p^{\prime}, q^{\prime} \in R, a \neq 0$, i. e.

$$
p=\frac{b e+(a d-b c) q}{a(2 e-c q)}, p^{\prime}=\frac{b e-a d q}{a(2 e-c q)}, q^{\prime}=\frac{e}{a q}, q=\frac{1}{2}\left(\frac{c}{a} \pm \sqrt{\frac{c^{2}-4 a e}{a^{2}}}\right), c^{2} \geq 4 a e .
$$

Note that (43) has four real roots

$$
u_{1,2}=\frac{1}{2}\left(-p \pm \sqrt{p^{2}-4 q}\right), u_{3,4}=\frac{1}{2}\left(-p^{\prime} \pm \sqrt{\left(p^{\prime}\right)^{2}-4 q^{\prime}}\right)
$$

provided that

$$
p^{2}-4 q \geq 0 \text { and }\left(p^{\prime}\right)^{2}-4 q^{\prime} \geq 0 .
$$

Assume that $p \neq p^{\prime}$. By change of variables

$$
z=\frac{\mu t+\nu}{t+1}, d z=\frac{\mu-\nu}{(t+1)^{2}} d t
$$


in (42), we deduce that

$$
L_{0}=\frac{\sqrt{2}}{2} \int_{u_{1}}^{u_{2}} \frac{d z}{\sqrt{R(z)}}=A \int_{\frac{\mu u_{1}+\nu}{u_{1}+1}}^{\frac{\mu u_{2}+\nu}{u_{2}+1}} \frac{d t}{\sqrt{\left(1+k_{1} t^{2}\right)\left(1+k_{2} t^{2}\right)}}
$$

where

$$
\begin{aligned}
& A=\frac{\sqrt{2}}{2} \frac{\mu-\nu}{\sqrt{a\left(\nu^{2}+p \nu+q\right)\left(\nu^{2}+p^{\prime} \nu+q^{\prime}\right)}}, \\
& k_{1}=\frac{\mu^{2}+p \mu+q}{\nu^{2}+p \nu+q}, k_{2}=\frac{\mu^{2}+p^{\prime} \mu+q^{\prime}}{\nu^{2}+p^{\prime} \nu+q^{\prime}} .
\end{aligned}
$$

Here, $\mu$ and $\nu$ can be found from equations

$$
p(\mu+\nu)+2(q+\mu \nu)=0, \quad p^{\prime}(\mu+\nu)+2\left(q^{\prime}+\mu \nu\right)=0,
$$

hence

$$
\nu=-\frac{q^{\prime}-q}{p-p^{\prime}} \pm \sqrt{\left(\frac{q^{\prime}-q}{p-p^{\prime}}\right)^{2}-\frac{p q^{\prime}-p^{\prime} q}{p^{\prime}-p}}, \mu=-\frac{q^{\prime}-q}{p-p^{\prime}} \mp \sqrt{\left(\frac{q^{\prime}-q}{p-p^{\prime}}\right)^{2}-\frac{p q^{\prime}-p^{\prime} q}{p^{\prime}-p}} .
$$

If (43) has four real roots then $k_{i}<0$. By change of variables $t=\left(-k_{1}\right)^{-1 / 2} \sin \varphi$ in $(45)$, we deduce that

$$
L_{0}=\tilde{A} \int_{\varphi_{1}}^{\varphi_{2}} \frac{d \varphi}{\sqrt{1-k_{3} \sin ^{2} \varphi}}
$$

where

$$
\tilde{A}=\frac{A}{\sqrt{-k_{1}}}, k_{3}=\frac{k_{2}}{k_{1}}>0, \varphi_{i}=\arcsin \left(\frac{\sqrt{-k_{1}}\left(\mu u_{i}+\nu\right)}{u_{i}+1}\right) .
$$

In particular, if (43) has only two real roots then $k_{1}<0$ and $k_{2}>0$ or $k_{1}>0$ and $k_{2}<0$. Moreover, if $a>0$ then the problem has no solutions therefore we have to consider the case $a<0$ only. Assume that $k_{1}<0$. Then we obtain (48) with $k_{3}<0$.

In the case $p=p^{\prime}$, by change of variables $z=t-\frac{p}{2}$ in (42), we deduce that

$$
L_{0}=\frac{\sqrt{2}}{2} \int_{u_{1}}^{u_{2}} \frac{d z}{\sqrt{R(z)}}=\hat{A} \int_{u_{1}-\frac{p}{2}}^{u_{2}-\frac{p}{2}} \frac{d t}{\sqrt{a\left(1-\hat{k}_{1} t^{2}\right)\left(1-\hat{k}_{2} t^{2}\right)}}
$$

where

$$
\hat{A}=\frac{2 \sqrt{2}}{\sqrt{\left(p^{2}-4 q\right)\left(p^{2}-4 q^{\prime}\right)}}, \hat{k}_{1}=\frac{4}{p^{2}-4 q}>0, \hat{k}_{2}=\frac{4}{p^{2}-4 q^{\prime}}>0 .
$$

By change of variables $t=\left(\hat{k}_{1}\right)^{-1 / 2} \sin \varphi$ in (49), we find that

$$
L_{0}=\bar{A} \int_{\hat{\varphi}_{1}}^{\hat{\varphi}_{2}} \frac{d \varphi}{\sqrt{a\left(1-\hat{k}_{3} \sin ^{2} \varphi\right)}}
$$


where

$$
\bar{A}=\frac{A}{\sqrt{\hat{k}_{1}}}, \hat{k}_{3}=\frac{\hat{k}_{2}}{\hat{k}_{1}}>0, \hat{\varphi}_{i}=\arcsin \left(\sqrt{\hat{k}_{1}}\left(u_{i}-p / 2\right)\right) .
$$

Next, we consider the special cases $p^{2}=4 q$ or $\left(p^{\prime}\right)^{2}=4 q^{\prime}$. For example, if $\left(p^{\prime}\right)^{2}=4 q^{\prime}$ then

$$
R(u)=a\left(u-\frac{-p-\sqrt{p^{2}-4 q}}{2}\right)\left(u-\frac{-p+\sqrt{p^{2}-4 q}}{2}\right)\left(u+\frac{p^{\prime}}{2}\right)^{2} .
$$

If $a<0$ then two cases are possible:

$$
\begin{aligned}
& \text { (i) }-\frac{p^{\prime}}{2} \in\left(\frac{-p-\sqrt{p^{2}-4 q}}{2}, \frac{-p+\sqrt{p^{2}-4 q}}{2}\right), \\
& \text { (ii) }-\frac{p^{\prime}}{2} \notin\left(\frac{-p-\sqrt{p^{2}-4 q}}{2}, \frac{-p+\sqrt{p^{2}-4 q}}{2}\right) .
\end{aligned}
$$

In the first case (i), we find that $L_{0}=\infty$. In the second case (ii), we have (48). On the other hand, if $a>0$ then the problem has no solution in the case (i) but for the case (ii) we have $L_{0}=\infty$.

In particular, if $p^{2}=4 q$ and $\left(p^{\prime}\right)^{2}=4 q^{\prime}$ then

$$
R(u)=a\left(u+\frac{p}{2}\right)^{2}\left(u+\frac{p^{\prime}}{2}\right)^{2} .
$$

Obviously, if $a<0$ then the problem has no nontrivial solutions but if $a>0$ then $L_{0}=\infty$.

\section{References / Литература}

1. Cahn J.W., Hilliard J.E. Free Energy of a Nonuniform System, I. Interfacial Free Energy. The Journal of Chemical Physics, 1958, vol. 28, pp. 258-267. DOI: 10.1063/1.1744102

2. Carr J., Gurtin M.E., Slemrod M. Structured Phase Transition on a Finite Interval. Archive for Rational Mechanics and Analysis, 1984, vol. 86, pp. 317-351. DOI: 10.1007/BF00280031

3. Fife P.C., Penrose O. Interfaced Dynamics for Thermodinamically Consistent Phase-Field Models with Nonconcerved Order Parameter. Electronic Journal of Differential Equations, 1995, vol. 16, pp. 1-49.

4. Grinfeld M., Novick-Cohen A. Counting Stationary Solutions of the Cahn - Hilliard Equation by Transversality Arguments. Proceedings of the Royal Society of Edinburgh: Section A Mathematics, 1995, vol. 125, no. 2, pp. 351-370. DOI: 10.1017/S0308210500028079

5. Grinfeld M., Novick-Cohen A. The Viscous Cahn-Hilliard Equation: Morse Decomposition and Structure of the Global Attractor. Transactions of the American Mathematical Society 6, 1999, vol. 351, no. 6, pp. 2375-2406.

6. Provatas N., Elder K. Phase-Field Methods in Materials Science and Engineering. Weinheim, Wiley-VCH, 2010. 312 p.

7. Novick-Cohen A., Peletier L.A. Steady States of the One-Dimensional Cahn - Hilliard Equation. Proceeding of the Royal Society of Royal of Edinburg, 1993, vol. 123A, pp. 1071-1098. DOI: $10.1017 / \mathrm{s} 0308210500029747$ 
8. Smoller J., Wasserman A. Global Bifurcation of Steady-State Solutions. Journal of Differential Equations, 1981, vol. 39, pp. 269-290. DOI: 10.1016/0022-0396(81)90077-2

Received February 28, 2016

УДК 517.912

DOI: $10.14529 / \mathrm{mmp} 160206$

\title{
СТАЦИОНАРНЫЕ РЕШЕНИЯ УРАВНЕНИЯ КАНА - ХИЛАРДА С ГРАНИЧНЫМ УСЛОВИЕМ НЕЙМАНА
}

\section{И.Б. Краснюк, Р.М. Таранеи, М. Чугунова}

\begin{abstract}
Исследована структура стационарного состояния одномерного уравнения Кана Хилларда в сочетании с граничными условиями Неймана. Здесь свободная энергия задается полиномом четвертого порядка. Была построена диаграмма бифуркации существования и единственности монотонных решений этой задачи. А именно, найдена длина интервала, на котором решение монотонно возрастает или убывает и имеет один нуль для некоторых фиксированных значений физических параметров. Под неоднозначностью понимается возможность существования более чем одного монотонного решения для некоторых значений физических параметров.

Ключевые слова: уравнение Кана - Хиларда; граничное условие Неймана; устойчивые состояния.
\end{abstract}

Игорь Борисович Краснюк, кандидат физико-математических наук, старший научный сотрудник, Институт прикладной математики и механики НАН Украины (г. Донецк, Украина), krasnjukigr@rambler.ru.

Роман Михайлович Таранец, кандидат физико-математических наук, научный сотрудник, Калифорнийский университет (г. Лос-Анджелес, Соединенные Штаты Америки), taranets_r@yahoo.com.

Марина Васильевна Чугунова, профессор, Клермонтский университет (г. Клермонт, Соединенные Штаты Америки), marina.chugunova@cgu.edu.

Поступила в редакиию 28 февраля 2016 г. 